\title{
Carbenicillin Potassium
}

National Cancer Institute

\section{Source}

National Cancer Institute. Carbenicillin Potassium. NCI Thesaurus. Code C76220.

The potassium salt of carbenicillin, a broad-spectrum, semisynthetic penicillin derivative with antibacterial activity. Carbenicillin binds to and inactivates penicillin-binding proteins (PBPs) located on the inner membrane of the bacterial cell wall, thereby preventing the cross-linkage of peptidoglycans, which are critical components of the bacterial cell wall. This leads to a weakening of the bacterial cell wall, eventually causing bacterial cell lysis. 